\title{
Ice Nucleation of Model Nanoplastics and Microplastics: A Novel Synthetic Protocol and the Influence of Particle Capping at Diverse Atmospheric Environments
}

\author{
Mainak Ganguly, ${ }^{1}$ Parisa A. Ariya*1,2 \\ ${ }^{1}$ Department of Atmospheric and Oceanic Sciences, McGill University, Montreal, Quebec H3A \\ 0B9, Canada \\ ${ }^{2}$ Department of Chemistry, McGill University, Montreal, Quebec H3A 0B8, Canada \\ Corresponding author: parisa.ariya@mcgill.ca
}

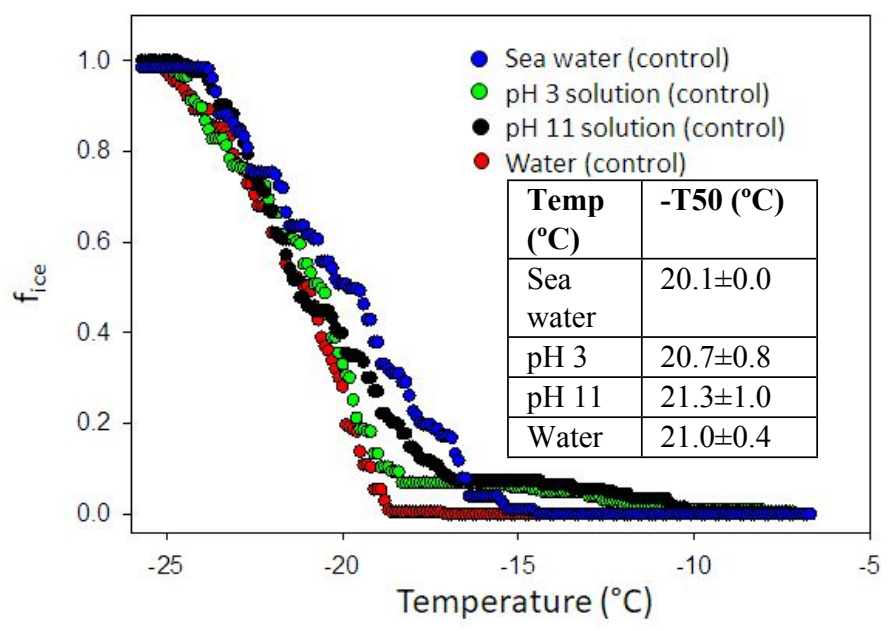

Figure S1: The freezing fraction and T50 values of the control solutions. 